Erschienen in: Stickel, Gerhard (Hrsg.): Sprache - Sprachwissenschaft - Öffentlichkeit.

Berlin/New York: de Gruyter, 1999. S. 73-84.

(Jahrbuch des Instituts für Deutsche Sprache 1998)

DOI: https://doi.org/10.1515/9783110622645-007

\title{
GERHARD VOIGT
}

\section{Die strukturbedingte Wissenschaftsferne des schulischen Sprachunterrichts ${ }^{1}$}

\begin{abstract}
Die Sprachwissenschaft antwortet auf Fragen, die die Öffentlichkeit nicht hat. Diese Konstellation, die vermutlich für jede Wissenschaft gilt, ist der Schule aus dem Verhältnis zwischen Lehrern und Schülern nicht unvertraut. Der Lehrer reagiert darauf damit, daß er die Schüler zu der Fragwürdigkeit von Tatbeständen und Meinungen hinzuführen versucht, bevor er im Unterricht mögliche Antworten entwickelt. Dieses Verhalten ist auf die Beziehung zwischen Sprachwissenschaft und Schule insoweit übertragbar, als die Sprachwissenschaft nicht nur selbstgenügsam als Wissende in sich selber ruhen darf, sondern künftigen Lehrern die Bedeutung ihrer Forschungen für ihren Beruf vermitteln muß. Das ist bisher oft nicht gelungen; Deutschlehrer interessieren sich weit mehr für Literatur als für Sprache. Es geht also nicht in erster Linie darum, die Fachinhalte für den Gebrauch von Lehrerstudenten aufzubereiten oder ihnen eine bestimmte Auswahl von sprachwissenschaftlichen Seminaren anzubieten, sondern darum, die Studenten für die deutsche Sprache zuerst einmal zu interessieren, vielleicht sogar zu begeistern. Das scheint möglich, wenn es gelingt, den Studenten die Erklärungskraft sprachwissenschaftlicher Theoriebildung für das Verständnis des alltäglichen mündlichen und schriftlichen Gebrauchs der deutsche Sprache vor Augen zu führen und ihnen so ein gegenüber ihrem vorwissenschaftlichen Verständnis neues Bild der deutschen Sprache - und erst dann ein Bild der germanistischen Linguistik - zu vermitteln.
\end{abstract}

„Wenn Ihr jetzt nicht endlich ruhig seid, dann mache ich mit Euch Grammatikunterricht!“

Natürlich wird kein vernünftiger Lehrer, und welcher Deutschlehrer wäre das nicht, einen solchen Satz äußern. Wenn er trotzdem immer mal wieder von dem einen Kollegen oder der anderen Kollegin kolportiert wird, dann wird ein alter Witz auf seine (oder ihre) Kosten gemacht, wenn es sich nicht sogar um gezielte üble Nachrede handelt. Gleichwohl hat

1 Für Anregungen und kritische Ermutigung danke ich Walther Dieckmann, Peter Eisenberg, Ingwer Paul und Jürgen Zeck. (Hilfreiche Hinweise gab auch die Leiterin des Landesamtes für Lehramtsprüfungen in Berlin, Frau OSchR Lau; ihr gilt ebenso mein Dank. 
auch der älteste Lehrerwitz wie jede Satire sein kleines Körnchen Wahrheit, an dem die Übertreibung sich kristallisierend ansetzen kann. In diesem Fall sind es sogar zwei.

Das erste, daß, so trivial es ist, Grammatikunterricht wie überhaupt Spracherziehung nach wie vor einen wesentlichen Bestandteil des Deutschunterrichts bilden. In der Öffentlichkeit werden gelegentlich andere Behauptungen aufgestellt: „Ächz, Würg, - eine Industrienation verlernt ihre Sprache" ${ }^{2}$ - sie treffen nicht zu.

Für ihren Unterricht brauchen angehende wie gestandene Lehrerinnen und Lehrer eine gründliche Ausbildung und eine fortlaufende Weiterbildung. Darauf werde ich im ersten, kürzeren Teil meines Vortrags eingehen.

Der Stachel des alten Lehrerwitzes liegt natürlich bei seinem zweiten, eigentlichen Wahrheitskörnchen. Das Verhältnis der Kolleginnen und Kollegen zum Sprachunterricht ist gespannt. Sie sehen seine Notwendigkeit zwar ein, fühlen sich ihm oft aber nicht oder nicht genügend gewachsen. So gelingt es ihnen nicht in dem gewünschten Maße, die Sprech- und Schreibfähigkeiten ihrer Schülerinnen und Schüler zu fördern, geschweige denn ein Interesse und gar Begeisterung für Aspekte der Sprache bei ihren Schülern zu wecken. Erst jüngst haben Martin Fix und Werner Knapp von der PH Ludwigsburg von einer Umfrage unter ihren Studienanfängerinnen berichtet, nach der 58\% der Befragten angaben, der Umfang des Grammatikunterrichts, den sie in ihrer Schulzeit erhielten, sei zu gering gewesen. Über $50 \%$ schätzten ihre Grammatikkenntnisse als nicht ausreichend ein, nur 5\% als gut. Dieses Selbstbild der Studentinnen halten Fix und Knapp für realistisch. „Teilweise kann nicht einmal der Stoff der Grammatik, der später laut Bildungsplan in derGrundschule vermittelt werden soll, als gesicherter Bestand angesehen werden." (Fix/Knapp 1998, S. 79, vgl. auch Ivo/Neuland 1991)

Warum das Verhältnis vieler Lehrerinnen und Lehrer zur Sprachwissenschaft so problematisch ist und welche Folgen das für ihre Erwartungen an die Sprachwissenschaft hat, darauf werde ich im zweiten Teil des Vortrags eingehen.

Die ursprüngliche Einladung zu diesem Vortrag stellte parallel zu den anderen heute hier am Rednerpult vertretenen Interessengruppen an die Schule als einem Teil der Öffentlichkeit die Frage „Was erwartet die Schule von der Sprachwissenschaft? " Diese Frage ist $m$. E. auf zweierlei Weise verstehbar, je nach dem, ob ich sie als Frage an die Schule als Institution oder als an den einzelnen Lehrer gerichtet verstehe. Verstehe ich sie dahin, welche Aus- und Fortbildung für Deutschlehrer die Schule als Institution von der Sprachwissenschaft erwartet, so ist die Erwartung enorm. Die sprachwissenschaftliche Ausbildung, könnte man allgemein sagen, muß

2 Der Spiegel 28/1984. Titel. 
den Lehrer in die Lage versetzen, die sprachlichen Schwierigkeiten seiner Schüler verstehen und analysieren zu können sowie Konzepte zu entwerfen, die sprachlichen Defizite der Schüler abzubauen, ihre kommunikativen Fähigkeiten im mündlichen wie im schriftlichen Bereich zu erweitern und ihnen eine Beschreibungssprache zu vermitteln, die den Schülern etwa im Falle von Mißverständnissen ermöglicht, an einem klärenden metasprachlichen Diskurs teilzunehmen. Es genügt ja nicht, daß der Lehrer als personifizierte standardsprachliche Norm die Äußerungen und das kommunikative Verhalten der Schüler als falsch oder richtig klassifiziert und alle Folgerungen daraus dem sprachlichen Ingenium seiner Schüler überläßt. Bei ihrem Schulabschluß sollen die Schüler, und zwar aller Schularten, nicht nur sprachlich sicher im Bereich der Standardsprache und etwa noch einer fach- oder berufsbezogenen Varietät sein, sondern mündlich und schriftlich, wenn schon nicht in allen, so doch in möglichst vielen kommunikativen Bereichen sich bewegen können. So gesehen müßte ein Lehrer nicht nur die standardsprachliche Norm und möglichst viele Varietäten aktiv beherrschen, sondern er müßte über alles oder wenigstens einen erheblichen Teil des sprachwissenschaftlichen Wissens der Gegenwart verfügen, um zu den skizzierten Analysen überhaupt fähig zu sein. Das ist eine sehr weitgehende Erwartung, die kaum zu befriedigen sein wird. Sie macht aber vielleicht das Gefühl drohender Überforderung, das einen Deutschlehrer beschleichen kann, verständlich.

Näher bestimmt wird der Umfang des nowendigen sprachwissenschaftlichen Wissens durch den Schultyp, an dem der Lehrer unterrichtet, sowie durch zusätzliche Lernbedingungen wie dem sozio-kulturellen Hintergrund der Schüler in dem jeweiligen Einzugsbereich, den Herkunftssprachen der Schüler, deren Muttersprache nicht Deutsch ist, oder den Besonderheiten der lokalen Umgangssprache. Hier löst sich schon die Möglichkeit zu einer allgemeinen Formulierung von Erwartungen an die Sprachwissenschaft in Teilbereiche auf, und ich kann nur aus der begrenzten Sicht des Schultyps, an dem ich unterrichte, wie des Bundeslandes, in dem ich arbeite, sprechen. ${ }^{3}$ Sicher ist die deutsche Grammatik als ein für alle Lehrer unumgänglicher Kernbereich der Ausbildung anzusehen. Ich kann mir auch keinen schulischen Bereich vorstellen, in dem nicht auch die Kenntnis der Normen und Regeln kommunikativer Prozesse oder des Erwerbs der gesprochenen wie der geschriebenen Sprache für den Lehrer unverzichtbar wäre, doch traue ich mir aus meiner Sicht eines Berliner Gymnasiallehrers schon darüber kein abschließendes Urteil mehr zu.

3 Gymnasialkollegen aus Bayern wie aus Nordrhein-Westfalen, die an der Mannheimer Tagung teilnahmen, bestätigten meine Situationsbeschreibung auch für ihre Bundesländer, so daß vielleicht über die Region hinaus eine gewisse Allgemeingeltung beansprucht werden kann. 
Darüber zu diskutieren und festzulegen, was das sprachwissenschaftliche Grundwissen einer Grundschullehrerin oder eines Studienrats ausmachen sollte, ist zudem der Aufgabenbereich eines eigenen Wissenschaftszweiges, der Fachdidaktik, oder wie Vertreter dieses Faches auch gerne sagen, der Fachunterrichtswissenschaft Deutsch (vgl. Ivo 1977, Menzel 1984). Sie schöpft ihre Legitimation nicht zuletzt gerade daraus, daß, weil die unmittelbare Übertragung fachwissenschaftlicher Ergebnisse auf die Schule zu höchst problematischen Ergebnissen führen kann, es einer vermittelnden Instanz bedarf, die Ergebnisse fachwissenschaftlicher Forschung auf die Aufgaben der Schule bezieht, die also etwa von den Bedürfnissen des jeweiligen Unterrichts her den Stand der Sprachwissenschaft kritisch mustert und für die Lehrer diejenigen Aspekte aufbereitet, die sie die sprachlichen Schwierigkeiten ihrer Schüler besser verstehen lassen, damit sie sie zu einer größeren Sprachsicherheit führen können. Sprachdidaktik ist die Brücke, die die Kluft zwischen Sprachwissenschaft und Schule zu schließen versucht, über die die Nachrichten aus der Sprachwissenschaft, wenn überhaupt, an die Schule gelangen. Die Sprachdidaktiker haben ausführliche und wohlbegründete Vorschläge gemacht, was obligatorisch im Studium von Lehrerstudenten sein, was im Unterricht der einzelnen Schularten behandelt werden sollte. Ihre Vorschläge beeinflussen die Neufassung von Rahmenplänen und Schulbüchern, sie entwerfen Unterrichtsmodelle in deutschdidaktischen Zeitschriften und wirken über all das auf die allmähliche Veränderung des Deutschunterrichts ein. ${ }^{4}$ Ihre im einzelnen unterschiedlichen Auffassungen darzustellen und die Reichweite ihrer Wirkung zu untersuchen, ist nicht meine Sache und hier nicht der Ort. Es genügt festzuhalten, daß der Institution Schule als einem Teil der Öffentlichkeit durchaus bewußt ist, daß sie der Bindung an die universitäre Sprachwissenschaft bedarf. ${ }^{5}$

4 In diesem Prozeß ist es vor allem die Fachdidaktik, die immer wieder auf Veränderungen drängt, wobei trotz negativer Erfahrungen Didaktiker manchmal nicht der Versuchung widerstehen können, eine interessante Überlegung aus der Fachwissenschaft allzu unmittelbar der Schule anzudienen. Retardierend wirkt sich dagegen häufig die Zulassungspraxis für Schulbücher aus. Dank der Kulturhoheit der Länder prüft jedes Bundesland jedes Schulbuch für sich noch einmal. Neue Schulbücher wollen zwar zumeist innovativ sein, aber da die Verlage die Schulbücher in möglichst vielen Bundesländern verkaufen wollen, gilt von vornherein nur der kleinste gemeinsame Nenner. Zudem werden die Zulassungsgutachten in der Regel von schon lange berufstätigen Deutschlehrern verfaßt, die die Schulbücher zum einen auf ihre Übereinstimmung mit dem jeweils geltenden Rahmenplan des Bundeslandes hin prüfen, zum anderen aber oft auch die Kompatibilität mit ihren eigenen Unterrichtsgewohnheiten zum Maßstab nehmen.

5 Es gibt daneben fraglos auch Aversionen der Schule gegenüber der Wissenschaft. So soll es selbst in jüngerer Zeit noch vorgekommen sein (so wird aus 
Mir scheint es wichtiger, Ihnen vorzutragen, warum trotz dieser Vermittlungshilfe der Sprachdidaktik die Frage „Was erwartet die Schule von der Sprachwissenschaft?", wenn sie an den einzelnen Lehrer gerichtet wird, wohl eher mit einem sehr skeptischen 'wenig' oder gar mit 'nichts' beantwortet werden würde. Warum haben, lautet dann die Frage, viele Deutschlehrer und über sie vermittelt, wie mir scheint, dann auch die Institution Schule ein derart distanziertes und oft auch ablehnendes Verhältnis zur Sprachwissenschaft? Die Frage mit den Verweisen auf die bekannte Lehrerfaulheit, deren Wissenschaftsfeindschaft und dem Verweis auf das Beharrungsvermögen aller Institutionen abzutun, würde zu kurz greifen. Das gibt es natürlich auch in der Schule wie in der Schulverwaltung, aber es geht $\mathrm{m}$. E. nicht um eine Schuldfrage, sondern um die Beschreibung von Barrieren individueller wie institutioneller Art. Diesen Aspekt der Frage nach dem Verhältnis zwischen Sprachwissenschaft und Schule habe ich mit der vielleicht etwas hölzernen Wendung „strukturbedingt“ in dem Titel dieses Vortrages ausdrücken wollen.

Wann kommt ein Deutschlehrer überhaupt in die Lage, danach zu fragen, was in der Sprachwissenschaft, was in der germanistischen Linguistik vorgeht? Idealerweise wären es die Schwierigkeiten und Fragen, die sich in seinem Untericht zeigen und von denen er das Gefühl hat, daß die Unterrichtsroutinen zu deren Bewältigung nicht mehr hinreichen. Aber das erste und oft nur schwer übersteigbare Hindernis ist die verfügbare Arbeitszeit. Diese Feststellung ist in höchstem Maße unoriginell, aber ich $\mathrm{mu} ß$ trotz des in der Öffentlichkeit verbreiteten Bildes darauf bestehen, $\mathrm{da} ß$ in einem so korrekturintensiven Fach wie Deutsch, insbesondere wenn es in einem in dieser Hinsicht vergleichbaren Fach etwa aus den neueren Philologien zusammen unterrichtet wird, die zur Weiterbildung verfügbare Zeit sehr gering bemessen ist. ${ }^{6}$ Diese Arbeitsbelastung ist im Lauf der Zeit auch nicht geringer geworden, sondern eher gestiegen. Der allgemeine Trend zur Verrechtlichung der Schule hat, um nur ein Beispiel zu nennen, die Durchführungsvorschriften für das schriftliche und mündliche Abitur so ausdifferenziert, daß im Verlauf meiner zwanzigjährigen schulischen Dienstzeit der Umfang des Erwartungshorizontes für die Aufga-

verschiedenen Bundesländern berichtet), daß der Eindruck von Lehrerausbildern, daß an der Universität keine richtige Germanistik mehr gelehrt (oder die Germanistik nicht mehr richtig gelehrt) werde, sich ihren Referendaren gegenüber in in dem Satz entlud: ,Vergessen Sie alles, was sie auf der Universität gelernt haben." (vgl. Mann 1993).

6 Der besonderen Korrekturbelastung der Deutschlehrer sowie der Lehrer für die neueren Fremdsprachen wird in Österreich wie in den deutschsprachigen Kantonen der Schweiz dadurch entsprochen, daß die Zahl ihrer Pflichtwochenstunden gegenüber der der anderen Fächern reduziert ist. 
benstellung im schriftlichen Abitur wie des Gutachtens für die einzelne Arbeit sich etwa verdoppelt haben.

Dieser Mangel an verfügbarer Arbeitszeit gilt für die Vorbereitung des Sprach- wie des Literaturunterrichts gleichermaßen. Nur ist im Gegensatz zum Literaturunterricht der Sprachunterricht „eine meist wenig geliebte Aufgabe" (Boueke 1984, S. 334). ${ }^{7}$ Dieser Feststellung ist auch heute nicht zu widersprechen. Es ist unstrittig, daß die meisten angehenden Deutschlehrer das Studium der Germanistik auf Grund ihres Interesses für die schöne Literatur aufnehmen. Daß sie sich auch mit Sprache, mit Schreiberziehung und mit Rechtschreibung beschäftigen müssen, wird ihnen zumeist erst im Laufe des Studiums deutlich, ohne daß deswegen ihr Interessenschwerpunkt sich veränderte. Ein Indiz für das primär literarische Interesse ist etwa die Wahl des Themas für die schriftliche Hausarbeit im Ersten Staatsexamen. Ich würde an dieser Stelle gerne exakte Zahlen angeben, wie viele der Prüfungskandidaten mit Germanistik als erstem Fach 1997 (und zum Vergleich 1987, 1977, 1967 etc.) in Berlin ein literaturbezogenes, wie viele ein sprachbezogenes Thema wählten (Mediävistik aufgeteilt). Aber solche Daten sind von der Schulverwaltung nicht erhoben worden. So kann ich auf Grund eigener Rückfragen an das Landesprüfungsamt und verschiedene Hochschullehrer Berliner Hochschulen nur schätzen, daß das Verhältnis wohl zwischen 20:1 und 30:1 liegen wird. Da innerhalb der Germanistik in Berlin der literaturwissenschaftliche Prüfungsteil obligatorisch ist, bei dem zweiten die Wahl zwischen Mediävistik und Linguistik freigestellt ist und der verbleibende dritte Teil durch das Grundstudium abgegolten wird, ist diese Gewichtung bereits vorgezeichnet. Sie zeigt, daß der Schwerpunkt innerhalb des Faches Deutsch auch von der Prüfungsbehörde letztlich nicht anders gesehen wird als von den Studierenden. Dieser Sachverhalt ist zu gewohnt, als daß er jemanden noch überraschen würde, dennoch müßte der fremde Blick über ihn stutzen, denn etwa in dem Berliner Rahmenplan für die Sekundarstufe I - und die Lehrpläne der anderen Bundesländer sehen in diesem Punkt nicht wesentlich anders aus - gilt von den drei Lernbereiche nur einer der $\mathrm{Be}$ schäftigung mit Literatur (,Literatur und Gebrauchstexte“), zwei dagegen gelten der Beschäftigung mit der Sprache (,,Sprachsystem und Sprachgebrauch" und ,Sprechen und Schreiben“). Dieselbe Tendenz zeigt sich auch bei der Wahl des Themas für die schriftliche Hausarbeit im Zweiten Staatsexamen. Hier wird wohl auf 20 literaturbezogene Themen ein sprachbezogenes Thema kommen. ${ }^{8}$ Das Verhältnis verschiebt sich deswegen

${ }^{7}$ Boueke bezog die Formulierung auf die „Reflexion über Sprache“ in der Sekundarstufe I, doch scheint mir die Verallgemeinerung zu "Sprachunterricht" sowohl Bouekes Intention wie der tatsächlichen Situation angemessen. 
etwas zugunsten der sprachbezogenen Themen, weil unter sie auch die Arbeiten etwa zur Aufsatzerziehung fallen, die in der fachwissenschaftlichen Ausbildung kein unmittelbares Pendant haben. Nicht anders sieht es bei den Lehrerausbildern (Fachseminarleitern) aus, bei denen es sich um erfahrenere Lehrer handelt, die neben ihrem normalen, zeitlich reduzierten Unterricht in dem anderen Teil ihrer Arbeitszeit die Referendare ausbilden. Von den 21 Fachseminarleitern für das Fach Deutsch im Land Berlin haben nach meiner Umfrage nur vier eine ihrer beiden Staatsexamenarbeiten über ein sprachliches Thema geschrieben, zwei der vier sogar beide.

Aus diesen Zahlen, die eine Situation wiedergeben, die sich nicht erst jüngst so entwickelt hat, sondern über Jahrzehnte - man darf vermuten: bundesweit - so existiert, darf natürlich nicht in einer Art Umkehrschluß gefolgert werden, daß die Deutschlehrer an dem Untericht im sprachlichen Bereich generell nicht interessiert oder für ihn kaum ausgebildet seien. Durch die konkreten Unterrichtserfahrungen ist bei manchen ein entsprechendes Interesse gewachsen, der eine oder andere Kollege wie Ausbilder hat Unterrichtsvorschläge zur Spracherziehung publiziert oder an Sprachbüchern mitgearbeitet, die Lehrerfortbildungen zum Grammatikunterricht und zur Rechtschreibung, zur Schreiberziehung im Verbundenen Sprachunterricht oder zur Sprachreflexion in der Sekundarstufe II hatten keinen Teilnehmermangel, wenn man auch über die Jahre feststellen konnte, daß es eine relativ feste, kleine Gemeinde von Kolleginnen und Kollegen ist, die man in diesen Fortbildungsveranstaltungen immer wieder antrifft. Die genannten Zahlenverhältnisse sollen nur darauf verweisen, daß ein Gleichgewicht zwischen literarischen und sprachlichen Kenntnissen und Interessen nicht erwartet werden darf und daß neben dem generellen Problem der Vermittlung zwischen Fachwissenschaft und Schule die Rezeption der Sprachwissenschaft mit besonderen Schwierigkeiten zu kämpfen hat. Wenn ein Deutschlehrer noch Zeit hat, einen Blick in Fachpublikationen zu werfen, so tut er es wohl am ehesten in dem Teilbereich seines Faches, mit dem er sich in seiner Ausbildung am intensivsten auseinandergesetzt hat, und das ist in aller Regel kein Thema aus der Sprachwissenschaft. So kann es auch nicht Wunder nehmen kann, daß Didaktiker, die den primärsprachlichen Unterricht beobachteten, ihm kein sehr positives Zeugnis ausgestellt haben (vgl. Boettcher 1994).

Woher kommt, muß man fragen, die ausschlaggebende Rolle der Literatur für das Studium und in dem Studium der Germanistik? Auch diese Frage ist nicht neu und es gibt eine ganze Menge Antworten: Weil die Studenten als Heranwachsende gerne lasen, weil sie in der Schule Erfolge mit

8 Auch diese Zahl wird von der Berliner Schulverwaltung nicht erhoben. 
ihren Interpretationsaufsätzen hatten, weil die Welt des Fiktionalen eine Alternative zu einer als unbefriedigend empfundenen Wirklichkeit bietet usw. Ich will sie nicht alle referieren, sondern nur eine hervorheben, weil sie die Art der sprachwissenschaftlichen Ausbildung betrifft: In ihrem Literaturstudium erfahren viele Studenten, so jedenfalls noch das Echo aus dem Mund vieler Kollegen, das Glücksgefuihl gelingender Interpretation. Ein Text, den man vielleicht unmittelbar zu verstehen meinte, erschließt sich dank der spezifischen Mittel der Literaturwissenschaft dem Leser auf eine neue Weise. Er liest, was er vorher nicht gelesen hat, er kommt, wie Brecht es einmal genannt hat, zu einem Genuß des Verstehens. Das scheint im sprachwissenschaftlichen Studium nicht oder in signifikantem Ausmaß weniger der Fall zu sein. In Gesprächen über sprachliche Fragen sind es nach meiner Erfahrung vor allem zwei Reaktionen, denen man begegnet. Die Erklärung eines sprachlichen Phänomens gilt als banal oder als zu kompliziert. Es liegt nahe, dieses Urteilsschema auf den traditionellen spracherzieherischen Maßstab 'falsch / richtig' zu beziehen. Alle Probleme, die nach ihm zu regeln sind, sind banal, alle, die es nicht sind, sind zu kompliziert. Die Untersuchung sprachlicher Fragen, würde das heißen, gilt noch immer vielen Lehrern lediglich als Anwendung bestehender, von anderen festgesetzter Normen und Regeln, nicht als ein hermeneutischer Prozeß. Doch ist natürlich auch bei einer Beschäftigung mit Sprache dieser Genuß des Verstehens möglich. Ich erinnere mich an eine Lehrerfortbildungsveranstaltung zur Rechtschreibdidaktik (nicht der Rechtschreibreform), in der ein Berliner Hochschullehrer zeigen konnte, daß auf der Grundlage einer Darstellung der Prinzipien der deutschen Rechtschreibung wie des Erwerbs der Schriftsprache die speziellen Schwierigkeiten eines rechtschreibschwachen, gleichwohl zu Recht gymnasialempfohlenen Schülers für die Kollegen plötzlich durchschaubar und unterrichtliche Fördermöglichkeiten erkennbar wurden.

Das insgesamt gespannte Verhältnis zwischen den Lehrern und der Sprachwissenschaft ist durch die jüngeren und jüngsten Erfahrungen mit den Versuchen der Sprachwissenschaft, unmittelbar in die Schule hineinzuwirken, verstärkt worden. Namentlich die 'Linguistisierung des Deutschunterrichts' vom Beginn der 70er Jahre bildet bis heute eine traumatische Erfahrung im Verhältnis der Schule zur Wissenschaft. Nicht nur, daß viele Deutschlehrer sich damals von außen überrollt und gegenüber den entsprechenden neuen Lehrplananforderungen unausgebildet und überfordert vorkamen, sondern auch die damals jüngeren Lehrer, die sich auf die Anforderungen einließen und in Zusatzkursen und viel Mehrarbeit den Anschluß an „die Wissenschaft“ zu gewinnen suchten, fühlten sich genasführt, als nach kurzem die 'neue Sprachwissenschaft' aus dem Deutschunterricht wieder eliminiert wurde. Geblieben ist zudem das Schlagwort von der „Linguistik im Deutschunterricht“, das, darauf wies 
Walther Dieckmann schon in der Diskussion der 70er Jahren hin, den Blick darauf verstellt, daß es im Schulunterricht um Sprache, nicht um Sprachwissenschaft gehen muß. Von einer 'neuen Literaturwissenschaft' im Deutschunterricht war trotz aller Häutungen des Faches nie die Rede, da nie in Zweifel stand, daß die Beschäftigung mit der schönen (oder nicht mehr schönen) Literatur der Gegenstand des Unterrichts ist. Es ist ein wenig peinlich diese alte, of besprochene und oft beklagte Geschichte noch einmal aufzugreifen. Aber man muß sich klarmachen, daß von den Vorgängen eine damals junge, innovationswillige Lehrergeneration betroffen war, die bis heute das Gros der (Deutsch-) Lehrerschaft stellt und noch für etwa 5-10 Jahre stellen wird.

Diese negativen Erfahrungen verfestigten sich auch institutionell. Im Laufe der späten 70er und der 80er Jahre verschwanden die sprachbezogenen Themen vor allem aus dem Untericht der gymnasialen Oberstufe. Schlaglichtartig mag dies die Entwicklung des Berliner Rahmenplans für die gymnasiale Oberstufe beleuchten. Waren es im „Vorläufigen Rahmenplan" von 1977 noch zwei Sprachsemester, "Sprachliche Kommunikationsformen“ (12.1) und „Sprachbetrachtung“ (12.2) neben zwei Literatursemestern, so stand in dem Rahmenplan von 1985 nur noch ein Semester „Sprachbetrachtung“ drei literarisch orientierten Semestern gegenüber. Im derzeit gültigen Berliner Rahmenplan von 1995 sind alle vier Semester literarisch orientiert. Dafür ist in jedem Semester ein sprachbezogener Unterichtsschwerpunkt obligatorisch, der historischen Anordnung der Lernstoffe gemäß an literarischen Denkmälern orientiert. Es liegt also in der Hand jedes einzelnen Lehrers, wie intensiv dieser sprachbezogene Unterrichtsschwerpunkt jeweils behandelt wird. ${ }^{9}$ Fragestellungen für die Semesterklausuren im Grund- wie im Leistungskurs beziehen sich jedenfalls nur äußerst selten auf das sprachliche Thema; sprachbezogene Abiturthemen kommen so gut wie gar nicht vor. - Als Marginalie sei nur angefügt, daß der erste gesamtdeutsche Schulgermanistentag nach der Vereinigung im Jahr 1992 sich mit keiner sprachwissenschaftlichen oder sprachdidaktischen Fragestellung beschäftigt hat.

Es besteht also die merkwürdige Situation, daß allenthalben in der Schule wie in der Öffentlichkeit der Rückgang der mündlichen wie schriftsprachlichen Fähigkeiten der Schüler beklagt wird, zugleich aber die schulische Auseinandersetzung mit ihm nicht forciert, wenn nicht gar reduziert wird. Möglicherweise bedeutet aber der nordrhein-westfälische Lehrplanentwurf für die gymnasiale Oberstufe von 1997 eine Trendwen-

9 Es charakterisiert die schulische Situation, daß Einwände gegen die weitere Reduktion des Sprachunterrichts in der Sekundarstufe II im Rahmenplan von 1995 von nur einer kleinen Handvoll der Deutschfachkonferenzen der 127 Berliner Gymnasien erhoben wurde. 
de: In ihm wird die Fortsetzung der Ausbildung im „Sprechen“ und „Schreiben“ aus der Sekundarstufe I sowie die „Reflexion über Sprache“ in der gymnasialen Oberstufe wieder deutlich gewichtet (vgl. Landesinstitut 1997, S. 6-11, 15-17). Vielleicht wirkt sich bei dieser Neukonzeption schon aus, daß sich gerade im Bereich der Ausbildung in der deutschen Grammatik, soweit ich es beurteilen kann, die Situation an den Universitäten deutlich verbessert hat; in der Schule macht sich das vorläufig aber nur wenig bemerkbar, weil seit vielen Jahren kaum noch junge Deutschlehrer eingestellt werden.

Das Image der Sprachwissenschaft in der Schule hat auch ihr jüngster, nur der seinerzeitigen 'Linguistisierung des Deutschunterrichtes' vergleichbar autoritativer Auftritt nicht verbessert. Die Hinführung zur Neuregelung der deutschen Rechtschreibung traf zwar auf eine Deutschlehrerschaft, der zu großen Teilen die Mängel der alten Regelung bewußt war, und sie war auch organisatorisch besser vorbereitet. In den meisten Bundesländern gab es nicht nur Verwaltungsvorschriften, sondern Lehrerfortbildungsveranstaltungen, erläuternde Broschüren, Arbeits- und Unterrichtshilfen. Je länger aber die Debatten um diese Neuregelung dauern, um so mehr verstärkt sich der Eindruck, daß der Vereinfachungswille der Reformer dem komplizierteren, aber eben wohl doch nicht willkürlichen Usus der Schreiber nicht gerecht geworden ist. Mit andern Worten, obwohl die Reform offensichtlich ganz wesentlich didaktisch motiviert ist, erweckt sie auch in der Schule allmählich den Eindruck, daß die Sprachwissenschaft ein weiteres Mal mit großem Nachdruck etwas noch Unausgereiftes, sprachwissenschaftlich noch nicht hinreichend Geklärtes vorschnell in die Schule getragen hat. ${ }^{10}$ Vielleicht hilft aber auch die anhaltende Debatte um die Neuregelung der Rechtschreibung, bei der es ja zu einem wesentlichen Teil um das Verstehen des Schreibusus und seiner Tendenzen geht, die Fixierung des Rechtschreibunterrichts wie des Sprachunterrichts überhaupt von der Dichotomie falsch oder richtig zu lösen. Ob zukünftig Probleme der Schreibnorm wie der sprachlichen Norm überhaupt im Untericht weniger rechthaberisch und mehr unter dem Aspekt ihrer jeweiligen Funktionalität behandelt werden, läßt sich noch nicht beurteilen; man darf also noch hoffen, daß der Reformversuch und seine Diskussion etwas Positives bewirken.

Welcher Schluß wäre für Sprachwissenschaftler, wenn Sie, wie ich hoffe, die Situation der Lehrer besser verstehen, zu ziehen? Ich habe nichts Einfaches anzubieten. Einfach wäre es, wenn ich hätte begründen können, daß eine gesprächsanalytische Ausbildung notwendig ist, eine phonetische aber nicht. Oder umgekehrt. Dann könnten die einen Hochschullehrer sich

$10 \mathrm{Vgl}$. etwa die Diskussionbeiträge in Eroms/Munske (1997). 
zurücklehnen und die anderen würden auch mal ein Seminar ausdrücklich für Lehramtsstudenten anbieten und vielleicht sogar einmal eine Lehrerfortbildungsveranstaltung übernehmen. Es geht aber um etwas anderes. Es geht darum, daß Sie die Lehrerstudenten und auch die bereits im Beruf tätigen Lehrer für Ihr Fachgebiet gewinnen, ihnen zeigen, daß die Sprachwissenschaft ebensosehr helfen kann, die Sprache neu zu sehen, wie die Literaturwissenschaft die Literatur.

Dabei geht es nicht um einen Konkurrenzkampf zwischen Sprach- und Literaturwissenschaftlern um die fachliche Neigung der Studenten. Im Gegenteil. In den Lehrplänen aller Bundesländer ist in der einen oder anderen Form vom Deutschunterricht als „Verbundenem Sprachunterricht", „Integriertem Deutschunterricht" o. ä. die Rede. Gemeint ist damit die Verpflichtung des Lehrers Literatur- und Sprachunterricht aufeinander zu beziehen, gerade nicht das eine oder das andere, sondern immer beides zusammen zu unterrichten. Das ist ein Anspruch an die (Gymnasial-) Lehrer, auf den sie in ihrem Studium kaum vorbereitet werden und dem gerecht zu werden äußerst schwierig ist. " Die fachwissenschaftliche Entwicklung ist aber eine ganz andere. Spätestens seit dem Rhedaer Memorandum (Memorandum 1969) steht die Einheit des Fachs Germanistik in Frage. ${ }^{12}$ Für die Auflösung der Germanistik in Sprach- und Literaturwissenschaft mag es wissenschaftstheoretisch gute Gründe geben. An den Vorwürfen der Literaturwissenschaft an die Sprachwissenschaft, daß sie illiterat sei, mag ja ebenso etwas dran sein wie an der Replik der Sprachwissenschaft, daß sich die Literaturwissenschaft für alles interessiere außer für die deutsche Sprache. ${ }^{13}$ Von der Schule her gesehen bedeutet diese Debatte zuerst einmal nur, daß, je stärker Literaturwissenschaft (zumal als Kulturwissenschaft) und Sprachwissenschaft auseinanderstreben, die Last der Einheit des Schulfachs, die völlig unumstritten ist, allein auf die Schultern der Lehrer gelegt wird.

Der Verweis auf das Konzept des Integrierten Grammatik- wie Deutschunterrichts meint nun aber nicht, daß für Lehrerstudenten die Trennung zwischen literaturwissenschaftlichem und sprachwissenschaftlichen Studium aufgehoben oder daß im sprachwissenschaftlichen Studium Unterricht vorbereitet werden soll, auch nicht, daß die germanistische Lingui-

1 Der Erfolg des Schulbuchs von Einecke (1991) zeigt, welches Bedürfnis hier vorhanden ist.

12 Zeck (1997, S. 84) verweist darauf, daß das Spannungsverhältnis zwischen den Gegenstandsbereichen der Germanistik nicht erst mit der modernen Linguistik in das Fach gekommen ist, sondern auch schon in den 50er und 60er Jahren inhaltliche Bezüge zwischen ihnen die Ausnahme waren.

13 Grosse (1997, S. 12) spricht im Verhältnis beider von „Gleichgültigkeit“, „Ignoranz" und "wechselseitigem Hochmut". 
stik ihre Lehre auf die Bedürfnisse der Lehrer zuschneiden soll ${ }^{14}$, gemeint ist nicht einmal, daß das Neueste aus der Sprachwissenschaft unmittelbar in die Lehrerausbildung aufgenommen werden soll; aus der Sicht der Schule dürfen die sprachwissenschaftlichen Theorien ruhig schon ein bißchen abgehangen sein. Nein, es geht letztlich darum - entschuldigen Sie, wenn der letzte Satz etwas pathetisch gerät - , daß Sie die Studenten in ihrem sprachwissenschaftlichen Studium für die Sprache begeistern; dann werden auch mehr Lehrer die sprachwissenschaftlichen Forschungen wahrnehmen und ihre Schüler sprachbewußter erziehen.

\section{Literatur}

Boettcher, Wolfgang (1994): Grammatikunterricht in Schule und Lehrerausbildung. In: Der Deutschunterricht 5/94, S. 8-31.

Boueke, Dietrich (1984): Reflexion über Sprache. In: Hopster, Norbert (Hg.) (1984): Handbuch „Deutsch“ Sekundarstufe I. Paderborn. S. 334-366.

Einecke, Günther (1991): Unterrichtsideen Integrierter Grammatikunterricht, Textproduktion und Grammatik, 5. - 10. Schuljahr. 2 Bdd. Stuttgart/Dresden.

Eroms, Hans-Werner / Munske, Horst Haider (Hgg.): Die Rechtschreibreform. Pro und Kontra. Berlin.

Fix, Martin / Knapp, Werner (1998): Linguistikeinführungen für Lehramtsstudierende. In: Didaktik Deutsch 4, S. 78-89.

Grosse, Siegfried (1997): Literaturwissenschaft und Linguistik. In: Mitteilungen des Deutschen Germanistenverbandes 3/1997. S. 12-18.

Ivo, Hubert (1977): Zur Wissenschaftlichkeit der Didaktik der deutschen Sprache und Literatur. Frankfurt am Main/Berlin/München.

Ivo, Hubert / Neuland, Eva (1991): Grammatisches Wissen. Skizze einer empirischen Untersuchung über Art, Umfang und Verteilung grammatischen Wissens (in der Bundesrepublik). In: Diskussion Deutsch 121, S. 437-493.

Landesinstitut für Schule und Weiterbildung [in Nordrhein-Westfalen] (1997): Gymnasiale Oberstufe. Deutsch. Lehrplanentwurf. Soest.

Memorandum zur Reform der Linguistik und der Literaturwisenschaft (1969). In: Kolbe, Jürgen (Hg.) (1969): Ansichten einer künftigen Germanistik. München. S. 217-220.

Mann, Reinhold (1993): „Lehrerbildung“. In: Deutsche Lehrerzeitung 44/93, S. 2.

Menzel, Wolfgang (1994): Fachunterrichtswissenschaft Deutsch - Bestimmung und Thesen. In: Ossner, Jakob / Melenk, Hartmut (Hgg.): Methoden der Sprachdidaktik. Methoden im Sprachunterricht. Beiträge des V. Symposions Deutschdidaktik. Ludwigsburg. S. 21-28.

Zeck, Jürgen (1997): Integration des germanistischen Grundstudiums - Themenbereich Textverstehen. In: Mitteilungen des Deutschen Germanistenverbandes 3/1997. S. 84-94.

14 Diese Vermittlung hat die Fachdidaktik zu leisten. Es wäre allerdings eine engere Kooperation zwischen Sprachwissenschaft, Sprachdidaktik und Lehrerausbildung der zweiten Phase dringend zu wünschen. 